Military Technical College Kobry El-Kobba, Cairo, Egypt

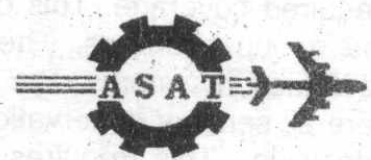

11-th International Conference on Aerospace Sciences \& Aviation Technology

\title{
ESTIMATION OF SENSORS RELIABILITIES USING NEURAL NETWORK AND FUZZY TECHNOLOGIES
}

\author{
Ashraf Mamdouh A. Aziz \\ Mohamed Hassan A. Azeem
}

\begin{abstract}
The sensors reliabilities in multisensor distributed decision systems with data fusion are the a priori statistical data needed to optimize the system it terms of higher detection probability. This paper proposes a new approach in data fusion systems to estimate the sensors reliabilities and to improve the system performance. The proposed approach is based on neural network and fuzzy logic technologies. Use of neural networks to learn system behavior seems to be a good way to solve the problem of the needed a priori statistical data in multisensor distributed detection systems with data fusion. Also, fuzzy logic has been proven very successful in solving problems in many areas where conventional model is either very difficult or inefficient/costly to implement. Use of fuzzy logic in multisensor distributed detection systems to determine uncertainty or confidence value (grade of membership function) for each hypothesis has the advantages of the soft decision approach. Combining the two technologies in multisensor distributed detection systems provides the benefits of both technologies. Thus using neural network and fuzzy logic technologies reduces the needed statistical data and improves system performance. The proposed approach does not require a priori statistical knowledge of the sensing process. The optimum fusion rule using the proposed approach is derived. The performance of the proposed approach is evaluated and compared to the performance of the hard-decision approach. The proposed approach provides detection probability improvement over a comparable hard-decision system and is able to correctly estimate the sensors reliabilities.
\end{abstract}

Key Words: Data fusion models - Distributed detection systems - Neural network Fuzzy logic.

\section{Introduction}

The problem of target detection with geographically distributed detection systems is of considerable importance in military surveillance systems [5], [10], [12]-[15]. The distributed sensors monitor a common volume and report their decisions/observations to a central processor for further processing. The fusion center combines the sensors 
decisions/observations into a final global decision. The basic goal of such multiple sensor systems is to improve system performance, increase the number of targets under consideration, and increase the required coverage. This can be achieved by integrating the information obtained from the various sensors. There are two major options for detection in multisensor distributed detection systems. The first option is centralized option (centralized detection system) where all sensors observations are transmitted to a central processor to derive a global decision. This requires transmission of all sensors observations without delay, which requires a large communication bandwidth. The second option is decentralized option (decentralized detection system with fusion) where the signal processing is distributed among the sensors and the fusion processor. The sensors are allowed to derive local decisions (hard decisions); then the fusion processor is responsible for integrating the received decisions from the various sensors into a final global decision .

The hard-decision approach does not provide any information to the fusion processor for signals below the decision threshold. In contrast to the hard decision approach, the soft decision approach allows the information to be integrated over a wide range of signal level. Several works has been reported to explore the fusion of hard and soft sensors decisions. Tenney and Sandell [8] have the pioneering effort in extending the Bayesian decision theory to the case of distributed sensors. Z. Chair et al. [9] derived the data fusion structure to be used at the fusion center which minimizes the overall probability of error. Thomopoulos et al. [11] derived the optimum fusion rule for the fusion of hard and semisoft (quality identified in a single bit) using Neyman Pearson criterion. ElAyadi et al. [13] proposed an algorithm for global optimization of distributed detection systems using Neyman Pearson strategy. E. Waltz [6] showed that the soft-decision has provided time and range improvements over a comparable hard-decision system .

This paper proposes a decision fusion approach based on fuzzy logic and neural network techniques. The proposed approach does not require prior statistical knowledge of the sensing process (probability density function and conditional probability matrix). The optimum fusion rule using the proposed approach is derived. The performance of the proposed approach is evaluated and compared to the performance of the hard-decision approach ( a fully informed approach). The proposed approach provides detection probability improvement over a comparable hard-decision system, thus it reduces the performance loss between the centralized and the decentralized (hard-decision) approaches. Furthermore, it is able to correctly estimate the false alarm and detection probabilities of the distributed sensors.

\section{Optimum Distributed Detection Systems With Data Fusion Models}

In a data fusion model, all sensor observations are transmitted to a central processor in order to derive a global decision $u_{0}$. No local decisions are made by the sensors. This is called centralized detection system. Under each hypothesis, the sensors observations have known joint probability densities $P\left(y_{1}, y_{2}, \ldots \ldots . y_{n} \mid H_{0}\right)$ and $P\left(y_{1}, y_{2}, \ldots \ldots y_{n} \mid H_{1}\right)$, where $y_{i}^{\prime} s, i=1,2, \ldots \ldots, n$ are random vectors representing the sensors observations. The crux of the centralized hypothesis-testing problem is to derive a decision strategy of the form: 


$$
u_{o}=\left\{\begin{array}{lll}
0 & H_{o} & \text { noise is present } \\
1 & H_{1} & \text { signal plus noise are present }
\end{array}\right.
$$

where $u_{0}$ depends on the observations. According to Neyman-Pearson criterion, it is required to find a decision strategy expressed as a density function $P\left(u_{0} \mid y_{1}, y_{2}, \ldots \ldots \ldots, y_{n}\right)$ which maximizes the global detection probability $(G D P)$ for a desired global false alarm probability (GFAP) where

$$
\begin{aligned}
& G F A P=\operatorname{Pr}\left\{u_{0}=1 \mid H_{0}\right\}, \\
& G D P=\operatorname{Pr}\left\{u_{0}=1 \mid H_{1}\right\} .
\end{aligned}
$$

The solution of the centralized problem is [5]:

(a) deterministic, so that the decision rule is a function

$$
\gamma\left(y_{1}, y_{2}, \ldots \ldots . y_{n}\right) \longrightarrow\{0,1\},
$$

where $u_{0}=i$ is interpreted as choosing $H_{i}$, and

(b) given by a Likelihood Ratio Test

$$
\operatorname{Lr}\left(y_{1}, y_{2}, \ldots \ldots y_{n}\right)=\left\{\begin{array}{l}
1 \text { ifLr }\left(y_{1}, y_{2}, \ldots \ldots . y_{n}\right)<t_{0} \\
0 \text { ifLr }\left(y_{1}, y_{2}, \ldots \ldots . y_{n}\right) \geq t_{0},
\end{array}\right.
$$

where

$$
\operatorname{Lr}\left(\mathrm{y}_{1}, \mathrm{y}_{2}, \ldots \ldots \ldots \mathrm{y}_{\mathrm{n}}\right)=\frac{\mathrm{P}\left(\mathrm{y}_{1}, \mathrm{y}_{2}, \ldots \ldots \ldots \mathrm{y}_{\mathrm{n}} \mid \mathrm{H}_{1}\right)}{\mathrm{P}\left(\mathrm{y}_{1}, \mathrm{y}_{2}, \ldots \ldots \ldots \mathrm{y}_{\mathrm{n}} \mid \mathrm{H}_{0}\right)} \text {, }
$$

(c) the threshold $t_{0}$ is determined according to the desired GFAP.

In case of decentralized detection system, it is allowed to the sensors to derive local decisions, then they are integrated in the data fusion center. This approach greatly reduces channel capacity for two reasons. First, a report of a decision is a simpler message than a sensor observation, and second, most observations need not be reported at all since they don't correspond to a detection. in this approach, a number of sensors $n$ receive and process the observations $y_{i}$ 's to generate the sensor decisions $u_{i}$, $i=1,2,3, \ldots \ldots, n$ with $u_{i}=1$ decide target present and $u_{i}=0$ decide target absent. The plot of the hard-decision versus the Likelihood ration test (LHRT) is shown in Fig. 1. The optimum data fusion structure using Neyman Pearson criterion is derived in [2], [6] and [8]. The individual decisions are weighted according to the detection and false alarm probabilities of each sensor $\left(p f_{i}, p d_{i}\right)$. The optimum data fusion structure is given by

$$
u_{0}=\left\{\begin{array}{l}
0 \text { if } \sum_{i=1}^{n} a_{i} u_{i}<\mathrm{T}_{0} \\
1 \text { if } \sum_{i=1}^{n} a_{i} u_{i} \geq \mathrm{T}_{0}
\end{array}\right.
$$

where

$$
a_{i}=\frac{p d_{i}\left(1-p f_{i}\right)}{p f_{i}\left(1-p d_{i}\right)},
$$

and the threshold $T_{0}$ is determined from the desired GFAP. When all sensors are similar and have a common operating point $(p f, p d)$, all the coefficients $a_{i}$ 's in (8) are equal, hence the optimum data fusion rule reduces to: 


$$
u_{0}=\left\{\begin{array}{l}
0 \text { if } \sum_{i=1}^{n} u_{i}<\mathrm{k} \\
1 \text { if } \sum_{i=1}^{n} u_{i} \geq \mathrm{k},
\end{array}\right.
$$

where $k$ is a positive integer. For $k=1,(9)$ reduces to a $O R$ fusion rule while for $k=n$, it becomes a $A N D$ fusion rule. The GFAP and GDP corresponding to (9) are given by:

$$
\begin{aligned}
& G F A P=\sum_{i=k}^{n} c_{i}^{n} p f^{i}(1-p f)^{n-i}, \\
& G D P=\sum_{i=k}^{n} c_{i}^{n} p d^{i}(1-p d)^{n-i},
\end{aligned}
$$

where

$$
c_{i}^{n}=\frac{n !}{i !(n-i) !}
$$

Thus, for every desired value of GFAP, there is an optimum integer $k$ that maximizes the GDP.

\section{Proposed Neural Network and Fuzzy Logic Approach}

We assume that there are $n$ detectors with statistically independent observations $y_{i}, i=1, \ldots \ldots, n$. Instead of reporting the sensors hard- decisions to the fusion center, each sensor is allowed to derive a soft-decision $\mu_{i}$ by defining a fuzzy set $A_{i}$ in $X$ as a set of ordered pairs :

$$
A_{i}=\left\{\left(x, \mu_{A i}(x)\right) \mid x \in X\right\}, i=1,2, \ldots ., n,
$$

where $\mu_{A i}(x)$ is called the membership function or grade of membership of $x$ in $A_{i}$ which maps the membership space $M$ in the interval [0,1]. If $M$ contains only the values of 0 and $1, A_{i}$ is nonfuzzy set and $\mu_{A i}(x)$ is identical to a nonfuzzy set (hard decision set). If $\mu_{A i}(x)$ is greater than 05 , the sensor will favor hypotheses $H_{1}$ and the corresponding hard decision will be $\mu_{i}=1$. If $\mu_{A i}(x)$ is less than 0.5 , the sensor is more likely to favor hypotheses $H_{0}$ and the corresponding hard decision is $u_{i}=0$. Thus the relation between the hard decision $u_{i}$ and the soft decision $\mu_{i}$ is then given by

$$
u_{i}=\left\{\begin{array}{l}
1 \text { if } \mu_{i} \geq 0.5 \\
0 \text { if } \mu_{i}<0.5 .
\end{array} .\right.
$$

In many cases, it is convenient to express the membership function of a fuzzy subset in terms of a standard function with adjustable parameters. Our human expertise contains two heuristics :

1 - as the difference between the Likelihood function and the threshold increases, the corresponding confidence (the grade of membership) of the decision increases and vise versa,

2- if the Likelihood function is equal to the threshold a value of 0.5 is a suitable value of the membership function in this case. According to heuristics 1 and 2 , a suitable membership function can be defined as: 


$$
\mu_{A}(x ; \alpha, \beta, \gamma)= \begin{cases}0 & \text { if } x \leq \alpha \\ 2\left[\frac{x-\alpha}{\gamma-\alpha}\right]^{2} & \text { if } \alpha \leq x \leq \beta \\ 1-2\left[\frac{x-\alpha}{\gamma-\alpha}\right]^{2} & \text { if } \beta \leq x \leq \gamma \\ 1 & \text { if } x \geq \gamma,\end{cases}
$$

where $x$ represents the likelihood ratio, $\beta$ represents the sensor threshold. The actual values of $\gamma$ and $\alpha$ depend on the expected signal range. The assigned membership function is shown in [8].

Let $\mathbf{u}$ be the vector formed of the sensors hard-decisions, i.e.,

$$
\mathbf{u}=\left(u_{1}, u_{2}, u_{3}, \ldots \ldots \ldots, u_{n}\right) \text {. }
$$

The Likelihood function of the fusion center is given by

$$
\operatorname{Lr}(\mathbf{u})=\frac{P\left(\mathbf{u} \mid H_{1}\right)}{P\left(\mathbf{u} \mid H_{0}\right)}=\frac{P\left(u_{1}, u_{2}, \ldots \ldots \ldots, u_{n} \mid H_{1}\right)}{P\left(u_{1}, u_{2}, \ldots \ldots \ldots, u_{n} \mid H_{0}\right)} .
$$

From the independent assumptions of the observations, we can write

$$
\operatorname{Lr}(\mathbf{u})=\frac{P\left(\mathbf{u} \mid H_{1}\right)}{P\left(\mathbf{u} \mid H_{0}\right)}=\prod_{i=1}^{n} \frac{P\left(u_{i} \mid H_{1}\right)}{P\left(u_{i} \mid H_{0}\right)} .
$$

Equivalently, we can write

$$
\begin{aligned}
& P\left(\mathbf{u} \mid H_{1}\right)=\prod_{S+} P\left(u_{i}=1 \mid H_{1}\right) \prod_{S-} P\left(u_{i}=0 \mid H_{1}\right), \\
& P\left(\mathbf{u} \mid H_{0}\right)=\prod_{S+} P\left(u_{i}=1 \mid H_{0}\right) \prod_{S-} P\left(u_{i}=0 \mid H_{0}\right),
\end{aligned}
$$

where $S+$ is the set of all $i$ such that $u_{i}=1\left(\mu_{i} \geq 0.5\right), S-$ is the set of all $i$ such that $u_{i}=0\left(\mu_{i}<0.5\right)$, and

$$
\begin{gathered}
P\left(u_{i}=1 \mid H_{1}\right)=P\left(\mu_{i} \geq 0.5 \mid H_{1}\right)=p d_{i}, \\
P\left(u_{i}=0 \mid H_{1}\right)=P\left(\mu_{i}<0.5 \mid H_{1}\right)=1-p d_{i}, \\
P\left(u_{i}=1 \mid H_{0}\right)=P\left(\mu_{i} \geq 0.5 \mid H_{0}\right)=p f_{i}, \\
P\left(u_{i}=0 \mid H_{0}\right)=P\left(\mu_{i}<0.5 \mid H_{0}\right)=1-p f_{i},
\end{gathered}
$$

and $p f_{i}$ and $p d_{i}$ are the false alarm and the detection probabilities of the $i^{\text {th }}$ sensor respectively. The corresponding log Likelihood ratio test is

$$
\log \operatorname{Lr}(\mathbf{u})=\sum_{s_{+}} \log \frac{p d_{i}}{p f_{i}}+\sum_{s_{-}} \log \frac{1-p d_{i}}{1-p f_{i}} .
$$

Therefore the data fusion rule can be expressed as

$$
u_{0}=\left\{\begin{array}{l}
0 \text { if } \sum_{i=1}^{n} b_{i} \mu_{i}<\log \lambda_{0} \\
1 \text { if } \sum_{i=1}^{n} b_{i} \mu_{i} \geq \log \lambda_{0},
\end{array}\right.
$$

where $\log \lambda_{0}$ is determined according to the desired GFAP and the optimum coefficients $b_{i}, i=1,2, \ldots, n$ are given by 


$$
b_{i}= \begin{cases}\log \frac{p d_{i}}{p f_{i}} & \text { if } \mu_{i} \geq 0.5 \\ \log \frac{1-p d_{i}}{1-p f_{i}} & \text { if } \mu_{i i}<0.5 .\end{cases}
$$

To estimate the coefficients $b_{i}$ 's, we use the competitive learning of the artificial neural networks. Artificial neural networks (or simply neural networks) try to mimic the human brain to be useful models for complicated engineering problems. The major advantage of a neural network is its ability to learn the underlying system behavior. One of the most used neural network is the Self-Organizing Map (SOM) developed by Kohonen [1]-[3]. As in the human brain, where learning is achieved through the process of chemical change in synaptic connections, learning in neural network is achieved through the synaptic weights. The synaptic weights vectors tend to approximate the density functions of the underlying processes [2].

The basic idea underlying the competitive learning is described by Kohonen [1] as follows: given statistical samples $\mathbf{X}(k)$, where $\mathrm{k}$ is the time index, and reference weights $\mathbf{m}_{i}(k), i=1,2, \ldots \ldots, m$, with initial values $\mathbf{m}_{i}(0)$, the weights are updated for the winning neurons according to the following relation (self-organized map [1]):

$$
\mathbf{m}_{i}(k+1)=\mathbf{m}_{i}(k)+\alpha(k)\left[\mathbf{X}(k)-\mathbf{m}_{\mathrm{i}}(k)\right], \quad 0<\alpha(k)<1 .
$$

Equation (28) describes the analytic expression of competitive learning. The optimum data fusion strategy, derived in section 3 and is shown in Fig.1, has the form of a typical one layer neural network. Approximation of the error probabilities of the sensors can be evaluated using (28) as:

$$
\begin{array}{r}
\hat{p}_{f i}(k+1)=\hat{p}_{f i}(k)+\alpha_{p f}(k)\left[\mu_{i}(k)-\hat{p}_{f i}(k)\right], \quad \text { if } \mu_{i}>0.5, u_{0}=0, \\
\hat{p}_{f i}(k+1)=\hat{p}_{f i}(k), \mu_{i}<0.5, u_{0}=0, \\
\hat{p}_{d i}(k+1)=\hat{p}_{d i}(k)+\alpha_{p d}(k)\left[\mu_{i}(k)-\hat{p}_{d i}(k)\right], \quad \text { if } \mu_{i}>0.5, u_{0}=1, \\
\hat{p}_{d i}(k+1)=\hat{p}_{d i}(k), \mu_{i}<0.5, u_{0}=1,
\end{array}
$$

where $\hat{p}_{f i}(k)$ and $\hat{p}_{d i}(k)$ are the estimates of false alarm and detection probabilities for the $i^{\text {th }}$ sensor at the $k^{\text {th }}$ time step, and $\alpha_{p f}(k)$ and $\alpha_{p d}(k)$ are suitable monotonically decreasing sequences (learning constants) of scalar-values weights, $0<\alpha_{p f}(k), \alpha_{p d}(k)<1$.

\section{Examples of Simulated Data Fusion Models}

By assuming the case of $n$-identical sensors with Gaussian distributed observations; i.e.

$$
P\left(y_{i} \mid H_{\theta}\right)=\frac{1}{\sqrt{2 \pi}} e^{-\left(y_{i}-\theta_{i}\right)^{2} / 2}, \theta=0,1, s_{i}>0
$$

the Neyman-Pearson test, utilizing all of the received observations $y_{i}$ 's in case of the centralized detection system, will have the form

$$
u_{0}=\left\{\begin{array}{l}
0 \text { if } \sum_{i=1}^{n} y_{i}<T \\
1 \text { if } \sum_{i=1}^{n} y_{i} \geq T .
\end{array}\right.
$$

To achieve a desired GFAP, a threshold of

$$
T=\sqrt{n} \phi^{-1}(G F A P),
$$


is needed at the fusion center, where the $\phi$ function is defined as

$$
\phi(x)=\int_{x}^{\infty} \frac{1}{\sqrt{2 \pi}} e^{-z^{2} / 2} d z .
$$

The corresponding GDP is given by

$$
G D P=\phi\left(\frac{T-n s}{\sqrt{n}}\right) .
$$

The decision rules of the sensors in case of the decentralized detection systems are given by

$$
u_{i}=\left\{\begin{array}{l}
0 \text { if } \operatorname{Lr}\left(y_{i}\right)=\frac{p\left(y_{i} \mid H_{1}\right)}{p\left(y_{i} \mid H_{0}\right)}<\eta_{i} \\
1 \text { if } \operatorname{Lr}\left(y_{i}\right)=\frac{p\left(y_{i} \mid H_{1}\right)}{p\left(y_{i} \mid H_{0}\right)} \geq \eta_{i} .
\end{array}\right.
$$

The corresponding false alarm and detection probabilities are

$$
\begin{aligned}
& p f=\phi\left(\eta_{i}\right), \\
& p d_{i}=\phi\left(\eta_{i}-s_{i}\right),
\end{aligned}
$$

where $\eta_{i}$ is the $i^{\text {th }}$ detector threshold and is determined according to the sensor false alarm probability. The common signal to noise ratio ( $S N R)$ of the sensors is evaluated as

where

$$
S N R=\frac{\left[E_{1}\left\{y_{i}\right\}-E_{0}\left\{y_{i}\right\}\right]^{2}}{\operatorname{Var}_{0}\left\{y_{i}\right\}}=s_{i}^{2}, i=1, \ldots ., n,
$$

$$
E_{\theta}\left\{y_{i}\right\}=E\left\{y_{i} \mid H_{\theta}\right\}, \theta=0,1 \text { and } \operatorname{Var}_{0}\left\{y_{i}\right\}=\operatorname{Var}\left\{y_{i} \mid H_{0}\right\} .
$$

The form of the membership of Eq..(15) is considered in the simulation. The parameter $\beta$, $\beta=(\alpha+\gamma) / 2$, is the crossover point. The values of $\alpha$ and $\gamma$ are taken to be

$$
\begin{aligned}
& \alpha=\beta-3 \sigma, \\
& \gamma=\beta+3 \sigma,
\end{aligned}
$$

where $\sigma$ is the standard deviation of the noise.

The fusion center performance is described as the receiver-operating characteristic $(R O C)$, which plots the detection probability versus the false alarm probability. Fig. 2 compares the global performance improvement in the centralized and the decentralized schemes in case of five identical sensors with Gaussian distributed observations and $0 d B$ per sensor observations. Fig. 3 also shows the common sensors ROC. The global performance improvement of data fusion systems (centralized or decentralized) over the individual sensor's $R O C$ is obvious. The performance loss due to the decentralized approach compared to the centralized approach is also obvious. Fig. 3 depicts the same plots using the proposed fuzzy decision approach. Comparing Fig. 2 and 3 , it is clear that the proposed fuzzy decision approach has better performance over the decentralized approach. Thus the fuzzy decision approach reduces the performance loss between the centralized

and the decentralized approaches. Figures 4 and 5 compare the estimated false alarm and detection probabilities (dashed curves) along with the true values (solid curves) for 
sensor 1 assuming $0 \mathrm{~dB}$ SNR and 0.5 sensor threshold. Due to the similarities of the figures only one operating point for one sensor is displayed to save space. As shown in Figures 4 and 5 , the estimated values according to the learning neural network with soft decisions gradually approach the true values.

\section{Conclusion}

in this paper, a new approach in data fusion systems, to estimate the sensors reliabilities and to improve the system performance, has been proposed. The proposed approach has the advantages of fuzzy and neural network technologies. It has been shown that the proposed approach has better performance than hard-decision approach, thus it reduces the performance loss between the decentralized and the centralized approaches. It has been shown that the distributed detection system can be realized as a single layer artificial Neural network and the false alarm and detection probabilities of the sensors (sensors reliabilities) can be estimated using the analytic expression of the competitive learning. The proposed approach is adaptive in that it does not require a prior knowledge of the statistics of the underlying processes. Simulation results show that the proposed approach is efficient and accurate.

\section{References}

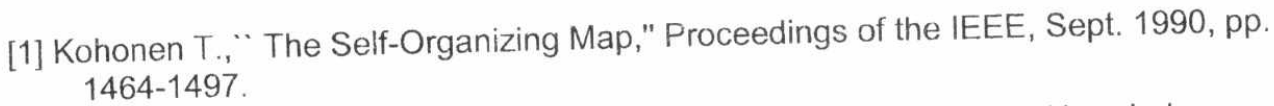

[2] Kohonen T., Foundations of Neural Networks, Fuzzy Systems, and Knowledge Engineering. Massachusetts Institute of Technology, 1996. [3] Kohonen T., ' Physiological Interpretation of the Self-Organizing Map Algorithm,"
Neural Network, Vol. 6, 1993, pp. 895-905.

[4] F. Russo and G. Ramponi, “'Fuzzy Methods for Multisensor Data Fusion", IEEE Transactions on Aerospace and Electronic Systems, Vol. 43, No-2, pp. 288-294, April 1994.

[5] Tao Li and I. Sethi, “ Optimal Multiple Level Decision Fusion With Distributed Sensors", IEEE Transactions on Aerospace and Electronic Systems, Vol. 29, No-4, pp. 1252-1259, Oct. 1993

[6] E. Waltz and J. Llinas, Multisensor Data Fusion. Norwood, MA: Artech House, 1990

[7] D. L. Hall, Mathematical Techniques in Multisensor Data Fusion. Norwood, MA: Artech House, 1992.

[8] Robert R Tenney and Nils R. Sandell, " Detection With Distributed Sensors", IEEE Transaction on Aerospace and Electronic Systems, Vol. AES-17, No. 4, pp. 501 510, July 1981.

[9] Z. Chair and P.K. Varshney, “ Optimal Data Fusion in Multiple-Sensor Detection Systems", IEEE Transactions on Aerospace and Electronic Systems , Vol. AES-22, No. 1, January 1986.

[10] R. Srinivasan, “ Distributed Radar Detection Theory", IEE Proc., Vol. 133, Pt. F. No. 1. pp. 55-60, Feb. 1986. 
Oopre tat.

[11] Thomopoulos, Ramanarayana and Bougoulias, " Optimal Decision Fusion in Multiple-Sensor Systems", IEEE Transactions on Aerospace and Electronic Systems, Vol. AES-23, No-5, pp. 644-652, 1987.

[12] E. Waltz and D. Buede, " Benefits of Soft Sensors and Probabilistic Fusion", Proceedings of SPIE, Vol. 1096, pp. 309-320, 1989.

[13] M. H. ElAyadi, Ashraf Mamdouh A: Aziz, and ElBasiony, “ An Algorithm for Global Optimization of Distributed Multiple-Sensor Detection Systems using NeymanPearson Strategy," Signal Processing, European International Journal, Vol. 51, NO. 2, pp. 137-145, June 1996.

[14] Ashraf Mamdouh A Aziz. " Multiple-Sensor Distributed Detection Systems With Data Fusion ," Proceedings of the 13th IEEE SP Society and EURASIP International Conference on Digital Signal Processing, Santorini, Greece, July 1997.

[15] Ashraf Mamdouh A. Aziz, Murali Tummala, and Roberto Cristi, “ Optimal Data Fusion Strategies Using Multiple-Sensor Detection Systems," Proceedings of the 31st IEEE Asilomar Conference on Signals, Systems, and Computers, California, USA, November 1997.

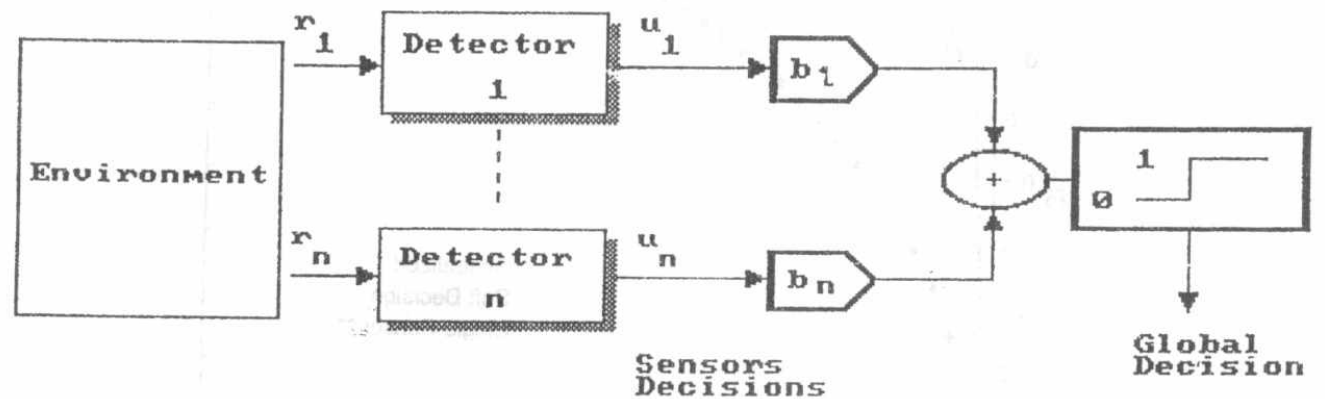

Fig.1 Distributed Detection System as One Layer Neural Network 

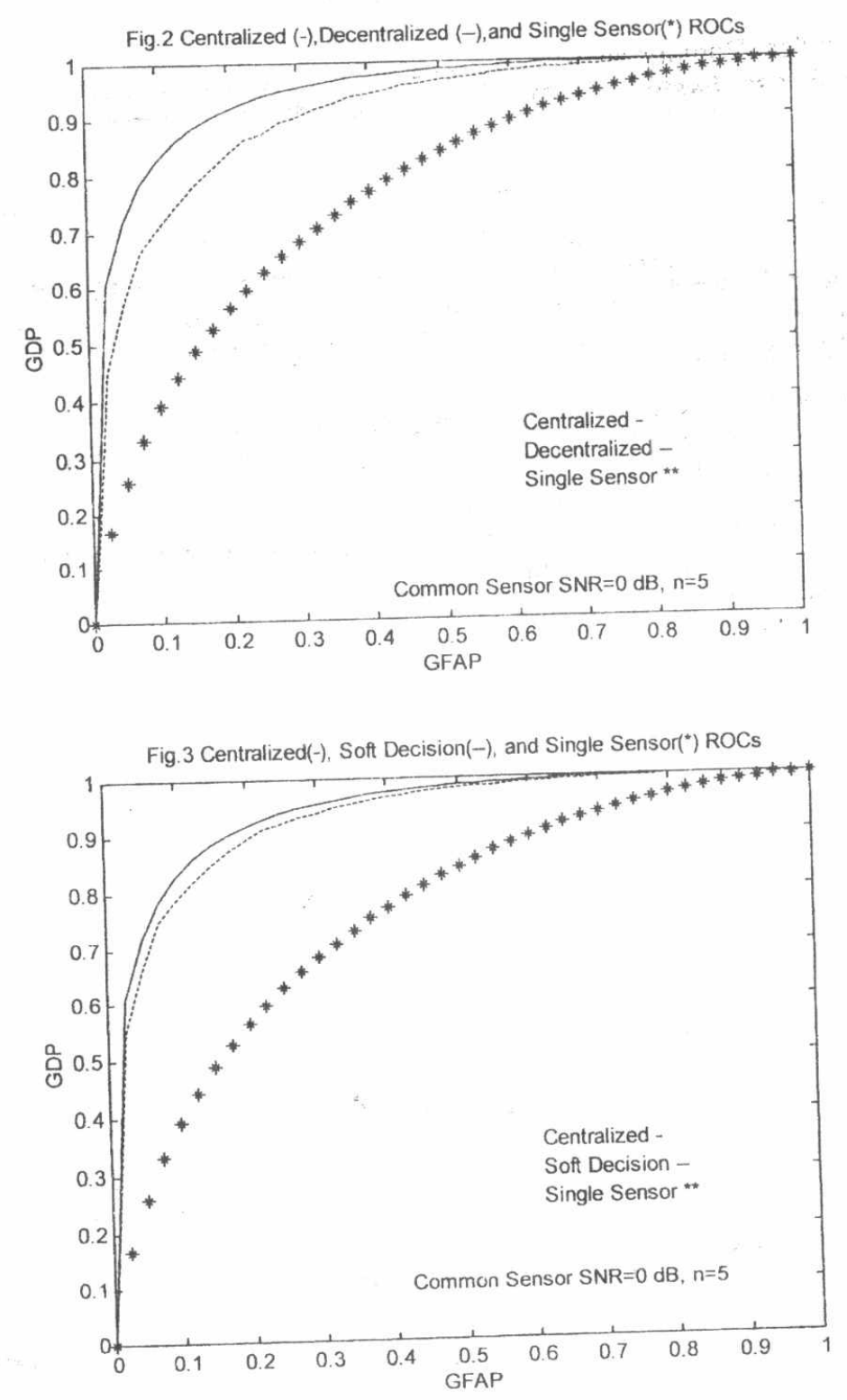

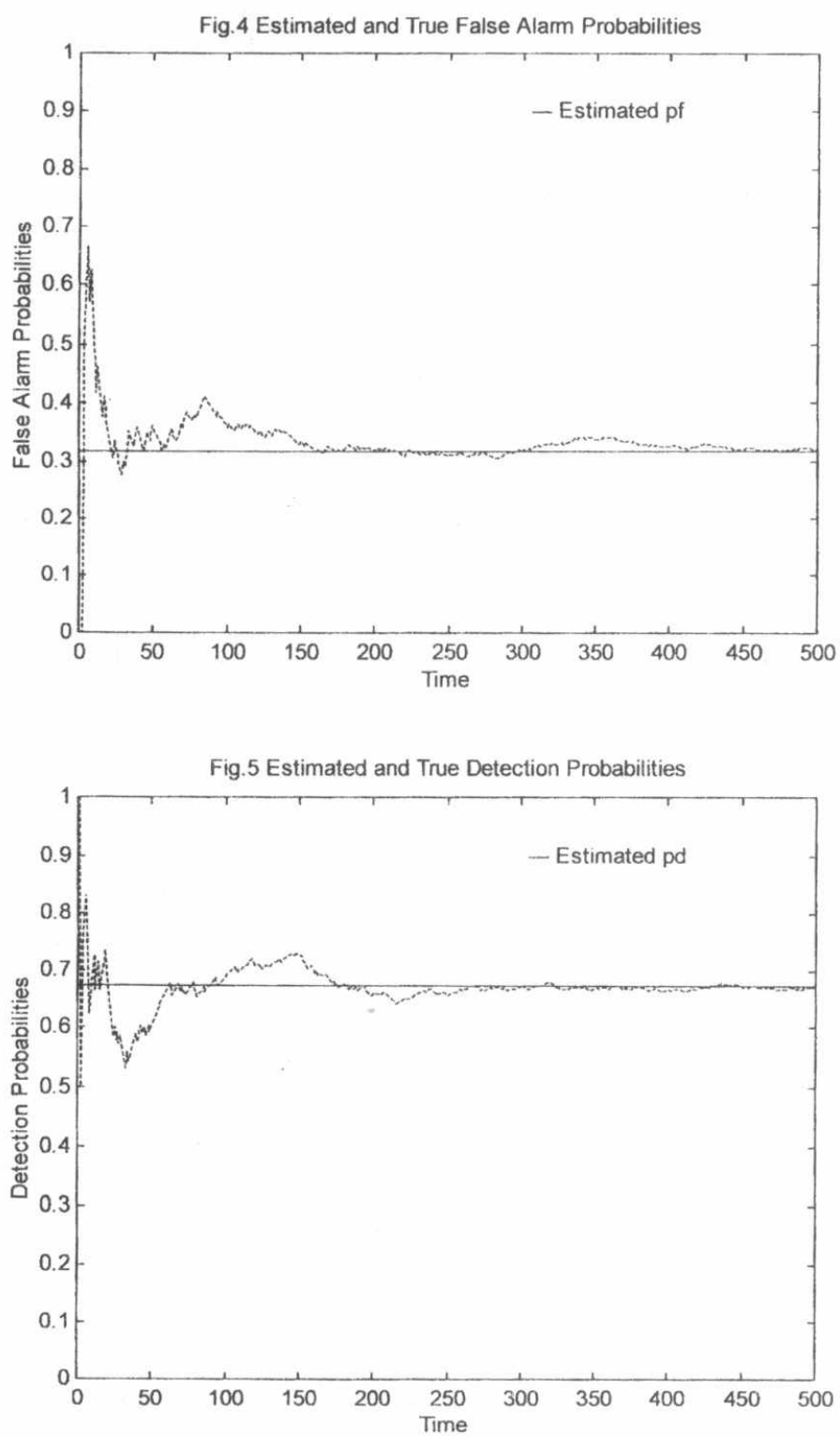